versa, particularly when interventional treatments are being planned.

\section{References}

1. Taşar M, Yetişer S, Taşar A, Uğurel S, Gönül E, et al. Congenital absence or hypoplasia of the carotid artery: radioclinical issues. Am J Otolaryngol. 2004;25:339-49.

2. Cinà CS, Althani H, Pasenau J, Abouzahr L. Kommerell's diverticulum and right-sided aortic arch: a cohort study and review of the literature. J Vasc Surg. 2004;39:131-9.
3. Kouchoukos NT, Masetti P. Aberrant subclavian artery and Kommerell aneurysm: surgical treatment with a standard approach. J Thorac Cardiovasc Surg. 2007;133: 888-92.

4. Wu JY, Chen HY, Shu CC, Yu CJ. Kommerell diverticulum, right-sided aorta, and left aberrant subclavian artery in a patient with dysphagia. J Thorac Cardiovasc Surg. In press.

5. Dinç H, Alioglu Z, Erdöl H, Ahmetoglu A. Agenesis of the internal carotid artery associated with aortic arch anomaly in a patient with congenital Horner's syndrome. Am J Neuroradiol. 2002;23:929-31.

\title{
Early thrombus in a HeartMate II left ventricular assist device: A potential cause of hemolysis and diagnostic dilemma
}

\author{
Castigliano M. Bhamidipati, DO, ${ }^{\mathrm{a}}$ Gorav Ailawadi, MD, ${ }^{\mathrm{a}}$ James Bergin, MD, ${ }^{\mathrm{b}}$ and John A. Kern, MD, \\ Charlottesville, Va
}

Thromboembolic events occur in $2 \%$ to $3 \%$ of patients receiving the HeartMate II (HMII) left ventricular assist device (LVAD) (Thoratec Corp, Pleasanton, Calif). Even though several surfaces of the HMII are textured and thromboresistant, long-term anticoagulation is indicated. ${ }^{1} \mathrm{We}$ report the case of an early postoperative distal inflowconduit thrombus resulting in hemolysis in a patient undergoing HMII placement and concomitant bioprosthetic aortic valve replacement (AVR).

\section{CLINICAL SUMMARY}

A 61-year-old woman with idiopathic cardiomyopathy, severe aortic stenosis, New York Heart Association class III heart failure, and a left ventricular ejection fraction of $15 \%$ to $20 \%$ (disproportionate to her aortic stenosis) was admitted for syncope and worsening dyspnea. Her comorbidities included obesity, diabetes, paroxysmal atrial fibrillation, previous stroke, and stage III chronic renal insufficiency. An AVR and HMII LVAD placement were performed, and the expectation was that the LVAD would serve as a bridge to recovery or a bridge to transplant. The LVAD insertion

\footnotetext{
From the Division of Thoracic and Cardiovascular Surgery, Department of Surgery, ${ }^{\text {a }}$ and Division of Cardiology, Department of Medicine, ${ }^{\mathrm{b}}$ University of Virginia School of Medicine, Charlottesville, Va.

Supported by National Institutes of Health Cardiovascular Surgery Research Training Grant T32/HL007849.

Disclosures: None.

Received for publication Sept 11, 2009; accepted for publication Sept 25, 2009; available ahead of print Nov 30, 2009.

Address for reprints: John A. Kern, MD, Associate Professor of Surgery, Medical Director of Non-Invasive Vascular Laboratory, Department of Surgery, Division of Thoracic and Cardiovascular Surgery, University of Virginia Health System, PO Box 800679, Charlottesville, VA 22908 (E-mail: jkern@virginia.edu).

J Thorac Cardiovasc Surg 2010;140:e7-8

0022-5223/\$0.00

Published by Elsevier Inc. on behalf of The American Association for Thoracic Surgery doi:10.1016/j.jtcvs.2009.09.046
}

and AVR proceeded uneventfully with satisfactory unloading of the left ventricle. Although fastidious surgical hemostasis was achieved, phytonadione, aminocaproic acid, and multiple blood product transfusions were necessary through the first 12 hours postoperatively; recombinant factor VIIa was not required. The patient was extubated within 24 hours and progressed well. Systemic anticoagulation with warfarin was initiated within 72 hours (Figure 1).

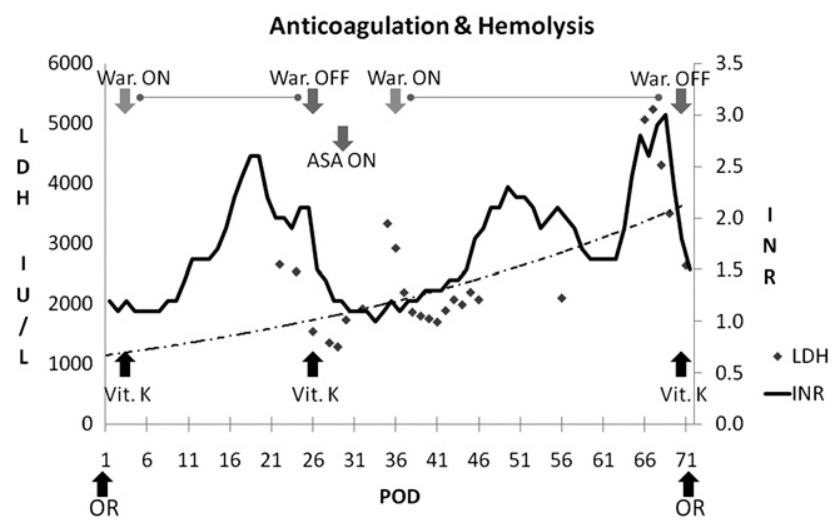

FIGURE 1. Anticoagulation and hemolysis timeline from HMII and AVR implantation through HMII pump replacement. Vitamin K (dark arrows) was administered on POD 1 because of suspected hemorrhage/excessive postoperative bleeding and on POD 26 because of evolving hematomas around the pump pocket site. Vitamin $\mathrm{K}$ was administered on POD 71 to reverse therapeutic anticoagulation in preparation for HMII replacement. Warfarin and ASA administration are identified by grayscale arrows with the INR trend (dark curve) consistent with warfarin administration. LDH activity (data points) remained significantly elevated (dashed regression trend) with the concomitant haptoglobin level less than $8 \mu \mathrm{mg} / \mathrm{dL}$ throughout. Taken together, they were suggestive of hemolytic anemia. Regression trend suggests LDH activity increased with lowered systemic anticoagulation. ASA, Aspirin; $A V R$, aortic valve replacement; $H M I I$, HeartMate II; $I N R$, international normalized ratio; $L D H$, lactate dehydrogenase; $O R$, operation; $P O D$, postoperative day; Vit, vitamin; War, warfarin. 


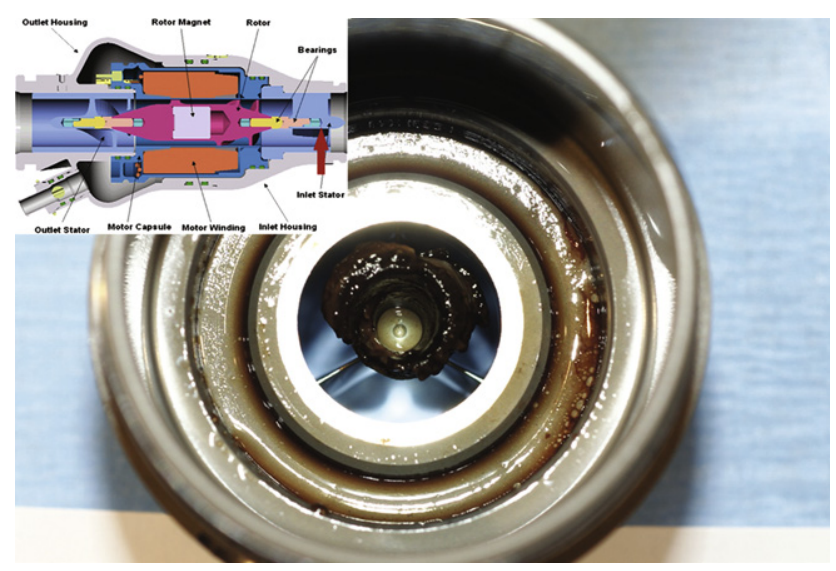

FIGURE 2. Thrombus at the distal end of the inflow-conduit as seen through the pump body of the HMII. Note the red arrow in the lateral profile of the HMII LVAD (inset). The inlet bearing cup is identified as the location of the distal inflow-conduit thrombus. Multiple LVAD studies showed normal function despite the presence of thrombus, suggesting current modalities may be inadequate to evaluate HMII LVAD pump body lesions. HMII, HeartMate II; $L V A D$, left ventricular assist device. (Image use approved by Thoratec Corp.)

The day before planned discharge (postoperative day 18), acute-onset hematuria and dyspnea developed. Transthoracic echocardiography showed occasional opening of the aortic valve with no paravalvular leak and normal flow into the LVAD. A large left pleural effusion subsequently developed, necessitating reintubation. Repeated red blood cell transfusions in response to decreasing hematocrit level led to the temporary cessation of warfarin. Transesophageal echocardiography with HMII rotor speed adjustments showed expected variations in left ventricular volume. Cardiac catheterization with left ventriculography confirmed what appeared to be normal, but perhaps slightly delayed flow through the LVAD. There appeared to be no inflow-conduit or outflowgraft patency limitations. She subsequently had jaundice, hemoglobinuria, and elevated lactate dehydrogenase (LDH). Hematologic studies, including von Willebrand factor, glucose-6-phosphate dehydrogenase, fibrinogen, haptoglobin, and D-dimer levels, suggested ongoing hemolysis. The LVAD rotor speed was reduced to attenuate any potential mechanical source. The HMII controller showed no change in pump power or differential pressure with a pulsatility index minimally above expectations.

Owing to ongoing hemolysis, a decision was made to replace the HMII. Intraoperatively, a thrombus on the inflow side of the bearing cup was identified (Figure 2). The HMII pump was replaced and the previously placed inflowconduit and outflow-graft were reattached. Systemic anticoagulation was initiated postoperatively, and an international normalized ratio of more than 2.0 was maintained. She had an uneventful postoperative course, was discharged home within 2 weeks of her second operation, and had made satisfactory progress at her 3-month follow-up visit.

\section{DISCUSSION}

Although thrombus formation is a known late complication of the HMII, ${ }^{2}$ early postoperative thrombus formation on the distal inflow-conduit has not been reported and, in our patient, was a unique cause of hemolysis. The most widely used anticoagulation protocol in patients receiving the HMII is that reported by the Texas Heart Institute. ${ }^{3}$ The decision to delay systemic anticoagulation until postoperative day 3 in our patient was based on postoperative bleeding, coagulopathy, and the low thromboembolic profile of the HMII. The use of phytonadione to correct coagulopathy in the face of chronic renal insufficiency challenges anticoagulation, with the early use of intravenous phytonadione having a putative delay in therapeutic systemic anticoagulation. The unclear source of hemolysis in the face of numerous false-negative LVAD interrogations compounded the difficult diagnosis.

Reportedly, von Willebrand factor-dependent platelet aggregation and related thrombocytopenia is another potential source of thrombus. ${ }^{4}$ On further review of the HMII pump, the thrombus around the inlet-bearing cup appeared as a formation of tissue and denatured blood that had undergone thermal degradation because of prolonged exposure to bearing heat. The thrombus demonstrated evidence of laminated layering, indicative of growth over an undetermined amount of time, presumably in the face of adequate anticoagulation. The size of the thrombus may have caused nonlaminar flow to the rotor and subsequent hemolysis.

Absolute anticoagulation requirements after HMII placement are unclear. One single-center analysis found the HMII to have an extremely low thromboembolic risk, allowing for less stringent anticoagulation requirements in certain patients. ${ }^{5}$ Those at high risk for bleeding can sometimes be monitored safely with either no or extremely low anticoagulation requirements. ${ }^{5}$ We have seen gastrointestinal bleeding after HMII implantation, requiring cessation of anticoagulation for 2 weeks while the bleeding was managed with no resultant thrombotic complications. Despite the low thromboembolic profile of the HMII, early thrombotic complications such as hemolysis can occur, posing a diagnostic challenge.

\section{References}

1. George I, Colley P, Russo MJ, Martens TP, Burke E, Oz MC, et al. Association of device surface and biomaterials with immunologic sensitization after mechanical support. J Thorac Cardiovasc Surg. Jun 2008;135:1372-9.

2. Meyer AL, Kuehn C, Weidemann J, Malehsa D, Bara C, Fischer S, et al. Thrombus formation in a HeartMate II left ventricular assist device. J Thorac Cardiovasc Surg. 2008;135:203-4.

3. Amir O, Bracey AW, Smart FW, Delgado RM 3rd, Shah N, Kar B. A successful anticoagulation protocol for the first HeartMate II implantation in the United States. Tex Heart Inst J. 2005;32(3):399-401.

4. Klovaite J, Gustafsson F, Mortensen SA, Sander K, Nielsen LB. Severely impaired von Willebrand factor-dependent platelet aggregation in patients with a continuous-flow left ventricular assist device (HeartMate II). J Am Coll Cardiol. Jun 2009;53(23):2162-7.

5. John R, Kamdar F, Liao K, Colvin-Adams M, Miller L, Joyce L, et al. Low thromboembolic risk for patients with the Heartmate II left ventricular assist device. J Thorac Cardiovasc Surg. 2008;136:1318-23. 